\title{
Molecular Cloning, Sequence Identification and Tissue Expression Profile of Three Novel Sheep (Ovis aries) Genes - BCKDHA, NAGA and HEXA
}

\author{
G. Y. LIU and S. Z.GAO*
}

Key Laboratory of Animal Nutrition and Feed of Yunnan Province, Yunnan Agricultural University, Kunming 650201, China

\begin{abstract}
The complete coding sequences of three sheep genes- BCKDHA, NAGA and HEXA were amplified using the reverse transcriptase polymerase chain reaction (RT-PCR), based on the conserved sequence information of the mouse or other mammals. The nucleotide sequences of these three genes revealed that the sheep BCKDHA gene encodes a protein of 313 amino acids which has high homology with the BCKDHA gene that encodes a protein of 447 amino acids that has high homology with the Branched chain keto acid dehydrogenase E1, alpha polypeptide (BCKDHA) of five species chimpanzee (93\%), human $(96 \%)$, crab-eating macaque $(93 \%)$, bovine $(98 \%)$ and mouse $(91 \%)$. The sheep NAGA gene encodes a protein of 411 amino acids that has high homology with the alpha-N-acetylgalactosaminidase (NAGA) of five species human $(85 \%)$, bovine $(94 \%)$, mouse $(91 \%)$, rat $(83 \%)$ and chicken $(74 \%)$. The sheep HEXA gene encodes a protein of 529 amino acids that has high homology with the hexosaminidase A(HEXA) of five species bovine (98\%), human (84\%), Bornean orangutan $(84 \%)$, rat $(80 \%)$ and mouse $(81 \%)$. Finally these three novel sheep genes were assigned to GeneIDs: 100145857, 100145858 and 100145856. The phylogenetic tree analysis revealed that the sheep BCKDHA, NAGA, and HEXA all have closer genetic relationships to the BCKDHA, NAGA, and HEXA of bovine. Tissue expression profile analysis was also carried out and results revealed that sheep BCKDHA, NAGA and HEXA genes were differentially expressed in tissues including muscle, heart, liver, fat, kidney, lung, small and large intestine. Our experiment is the first to establish the primary foundation for further research on these three sheep genes.
\end{abstract}

Key terms: sheep, BCKDHA, NAGA, HEXA, tissue expression.

\section{INTRODUCTION}

Branched chain keto acid dehydrogenase E1, alpha polypeptide (BCKDHA) encodes the E1-alpha subunit of the branched-chain alpha-keto acid (BCAA) dehydrogenase complex (BCKD; EC 1.2.4.4), an innermitochondrial enzyme complex that catalyzes the oxidative decarboxylation of the branched-chain alpha-ketoacids derived from isoleucine, leucine, and valine. Mutations of this gene have also been reported to be associated with maple syrup urine disease (Flaschker et al., 2007; Naik et al., 2004; Chinsky et al., 2004).

The Alpha-N-acetylgalactosaminidase (NAGA) gene encodes the lysosomal enzyme, which cleaves alpha-Nacetylgalactosaminyl moieties from glycoconjugates. Mutations in NAGA have been identified as the cause of Schindler disease types I and II (type II is also known as Kanzaki disease) (Chabás et al., 2007; Kanekura et al., 2005).

Hexosaminidase A (HEXA) is the alpha subunit of the lysosomal enzyme betahexosaminidase that, together with the cofactor GM2 activator protein, catalyzes the degradation of the ganglioside GM2, and other molecules containing terminal $\mathrm{N}$ acetyl hexosamines. Beta-hexosaminidase is composed of two subunits, alpha and beta, which are encoded by separate genes. Both beta-hexosaminidase alpha and beta

\footnotetext{
* Corresponding author. To whom correspondence should be addressed: liuyg4567@163.com
} 
subunits are members of a family 20 of glycosyl hydrolases. Mutations in the alpha or beta subunit genes lead to an accumulation of GM2 ganglioside in neurons and neurodegenerative disorders termed the GM2 gangliosidoses. Alpha subunit gene mutations lead to Tay-Sachs disease (GM2-gangliosidosis type I) (Karpati et al., 2004; McGinniss et al., 2002).

Based on the above, these three genes are associated with three important mutation diseases of humans and these diseases had been all found in another kind of mammal, the mouse (Pessoa-Pureur et al., 2007; Herrmann et al., 1998; Miklyaeva et al., 2004). These inherited diseases may also occur in sheep. If this is true, it is essential to isolate these three genes from sheep for these diseases are highly related or potentially related to sheep production. On the other hand, if these diseases also occur in sheep, sheep would be another important model to study these diseases in humans. However, until today the sheep BCKDHA, NAGA and HEXA genes have not yet been reported.

In the present work, we isolated the coding sequences of sheep BCKDHA, NAGA and HEXA genes based on the conserved sequence information of humans, mice or other mammals and highly homologous sheep EST sequence information, and subsequently performed the necessary sequence analyses and tissue expression profile analyses for these genes. These established the primary foundation of understanding of these three sheep genes.

\section{MATERIALS AND METHODS}

Samples collection, RNA extraction and first-strand cDNA synthesis

The tissue samples of liver, small intestine, large intestine, lung, muscle, fat, spleen, and kidney, were derived from three 120 days old Yunnan local sheep. Total RNA extraction and first-strand cDNA synthesis for these tissue samples were performed as the methods describe by Liu (2004).
Isolation of the sheep BCKDHA, NAGA and HEXA genes

The RT-PCR was performed to isolate these three sheep genes using the pooled cDNAs from different tissues above. The $25 \mu \mathrm{l}$ reaction system was: $2.0 \mu \mathrm{l}$ cDNA, $2.5 \mu \mathrm{l} 2$ $\mu \mathrm{M}$ mixed dNTPs, $2.5 \mu \mathrm{l} 10^{\prime} \mathrm{Taq}$ DNA polymerase buffer, $2.5 \mu \mathrm{l} 25 \mu \mathrm{M} \mathrm{MgCl}_{2}$, $2.0 \mu \mathrm{l} 10 \mu \mathrm{M}$ forward primer, $2.0 \mu \mathrm{l} 10 \mu \mathrm{M}$ reverse primer, 2.0 units of Taq DNA polymerase $(1 \mathrm{U} / 1 \mu \mathrm{l})$, and $9.5 \mu \mathrm{l}$ sterile water.

The primers for sheep BCKDHA gene isolation were designed based on the conserved CDS information from human, rat and mouse BCKDHA genes and the highly homologous sheep EST sequences: EE823419, EE827113 and EE770739. Similarly, the primers for sheep NAGA gene isolation were designed based on the conserved CDS information from human, rat and mouse NAGA genes and the highly homologous sheep EST sequences: DY493345, EE823664 and EE748803. The primers for sheep HEXA gene isolation were designed based on the conserved CDS information from human and mouse HEXA genes and the highly homologous sheep EST sequences: EE803104, EE829775, DY517999and EE750801. These primer sequences and their annealing temperature for RT-PCR reaction are described in Table 1.

These PCR products for sheep BCKDHA, NAGA and HEXA cDNAs were then cloned into PMD18-T vector and sequenced bidirectionally with the commercial fluorometric method. At least five independent clones were sequenced for every gene.

\section{RT-PCR for tissue expression profile analysis}

RT-PCR for tissue expression profile analysis was performed as previously described elsewhere (Liu et al., 2007). We selected the housekeeping gene $\beta$-actin (Accession no: NM_001009784) was performed as a positive control. The primers of sheep BCKDHA, NAGA and HEXA genes, which were used to perform the RTPCR for tissue expression profile analysis, 
were the same as the primers for isolation RT-PCR above. The PCR reactions were optimized for a number of cycles to ensure product intensity within the linear phase of amplification. The $25 \mu \mathrm{L}$ reaction system was: $1 \mu \mathrm{l}$ cDNA (100 ng/ $\mu \mathrm{l}), 5$ pmoles each oligonucleotide primer, $2.5 \mu \mathrm{l} 2 \mathrm{mmol} / 1 \mathrm{mixed}$ dNTPs, $2.5 \mu 110 \times$ Taq DNA polymerase buffer, $2.5 \mu l 25 \mathrm{mmol} / \mathrm{l} \mathrm{MgCl} 2,1.0$ units of Taq DNA polymerase, and finally added sterile water to a volume of $25 \mu \mathrm{l}$. The PCR program initially started with a $94^{\circ} \mathrm{C}$ denaturation for $4 \mathrm{~min}$, followed by 25 cycles of $94^{\circ} \mathrm{C} / 1 \mathrm{~min}, \mathrm{Ta}{ }^{\circ} \mathrm{C} / 1 \mathrm{~min}, 72^{\circ} \mathrm{C} / 1 \mathrm{~min}$, then $72^{\circ} \mathrm{C}$ extension for $10 \mathrm{~min}$, finally $4^{\circ} \mathrm{C}$ to terminate the reaction.

\section{Sequence analysis}

The cDNA sequence prediction was conducted using GenScan software (http:// genes.mit.edu/GENSCAN.html).The protein prediction and analysis were performed using the Conserved Domain Architecture Retrieval Tool of BLAST at the National Center for Biotechnology Information (NCBI) server (http://www.ncbi.nlm.nih.gov/BLAST) and the ClustalW software (http://www.ebi.ac.uk/ clustalw).

\section{RESULTS AND DISCUSSION}

RT-PCR results for sheep BCKDHA, $N A G A$, and HEXA genes

Through RT-PCR with pooled tissue cDNAs, for sheep BCKDHA, NAGA, and HEXA, the resulting PCR products were $1344 \mathrm{bp}, 1236 \mathrm{bp}$ and 1590bp.

Sequence analysis

The cDNA nucleotide sequence analysis using the BLAST software at NCBI server

TABLE 1

Primers for sheep BCKDHA, NAGA and HEXA isolation and annealing temperature.

\begin{tabular}{lll}
\hline Gene & Primer sequence & Ta $/{ }^{\circ} \mathrm{C}$ \\
\hline BCKDHA & $\begin{array}{l}\text { Forward 5' - ATGGCGTTGGCGGTGGCT -3 } \\
\text { Reverse:5' - TCACTTCTCAAAGTGGTCC-3' }\end{array}$ & 53 \\
NAGA & Forward :5' - ATGTTGCTGAAGACAGTGC -3 \\
& Reverse: 5' - TCACTGCGGGGGCGTCCCC -3 \\
HEXA & Forward 5' - ATGGCAGCCTCCACGCTC -3' \\
& Reverse:5' - TCAGGTTTGTTCAAACTCCAC-3' & 55 \\
$\beta$-actin & Forward: 5' - CTTGATGTCACGGACGATTT -3 \\
& Reverse: 5' - CACGGCATTGTCACCAACT -3' & 54 \\
\hline
\end{tabular}
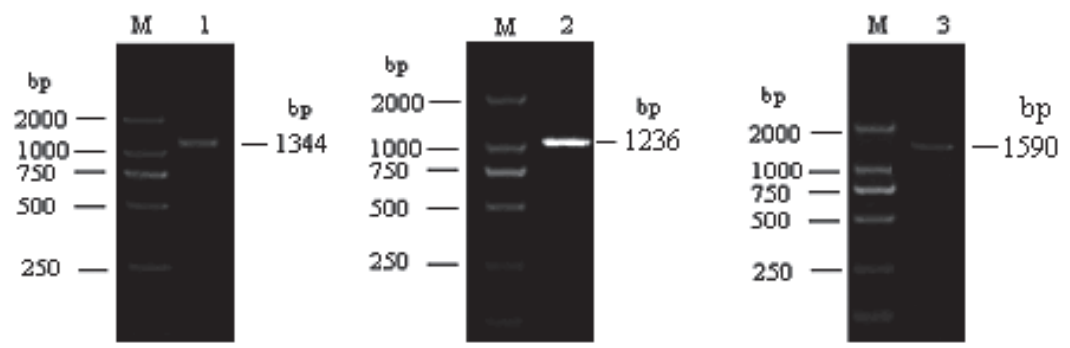

Fig. 1: RT-PCR results for sheep BCKDHA, NAGA, and HEXA. M, DL2000 DNA markers; 1, PCR product for sheep BCKDHA gene; 2, PCR product for sheep NAGA gene; 3, PCR product for sheep HEXA gene. 
Sheep

Bovine

Chimpanzee

Human

Crab-eating macaque

Mouse

\author{
Sheep \\ Bovine \\ Chimpanzee \\ Human \\ Crab-eating macaque \\ Mouse
}

Sheep

Bovine

Chimpanzee

Human

Crab-eating macaque Mouse

Sheep
Bovine
Chimpanzee
Human
Crab-eating macaque
Mouse

Sheep

Bovine

Chimpanzee

Human

Crab-eating macaque Mouse

Sheep

Bovine

Chimpanzee

Human

Crab-eating macaque Mouse

Sheep
Bovine
Chimpanzee
Human
Crab-eating macaque
Mouse
Sheep
Bovine
Chimpanzee
Human
Crab-eating macaque
Mouse

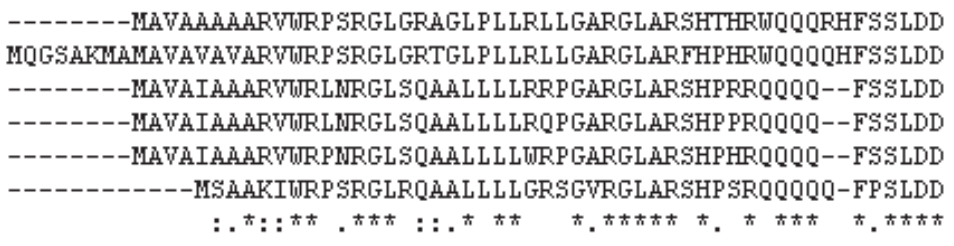
MQGSAKMAMAVAVAVARVURPSRGLGRTGL P L LRL L GARGLARFHPHRUQQQQHFSSLDD -------MAVA IAAARVIRLNRGLSQAAL LLLRRP GARGLARSHPRRQQQQ--FSSLDD -------MAVA IAAARVIRLNRGLSQAAL L L LRQP GARGLARSHP PRQQQQ--FSSLDD

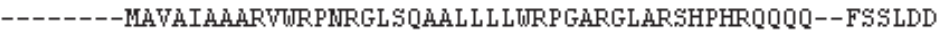
----------MSAAKIWRPSRGLRQABL L L L GRSGVRGLARSHP SRQQQQQ-FPSLDD

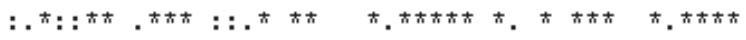

KPQFPGÄSAEFIDKLE FIQPNVISGIPIYRVMDRQGQIINPSED PHLPQEKVLKFYKSMT KPQFPGASAE FIDKLE FIQPNVISGIP IYRVMDRQGQIINP SED PHL PQEKVLKFYKSMT KPQFPGẢSAE FIDKLE FIQPNVISGIPIYRVNDRQGQIINPSED PHL PKEKVLKLYKSMT KPQFPGASAE FIDKLE FIQPNVISGIP IYRVMDRQGQIINP SED PHL PKEKVLKL YKSMT KPQFPGASAE FIDKLE FIQPNVISGIP IYRVMDRQGQIINPSED PHL PKEKVLKL YKSMT KPQFPGASAE FVDKLEFIQPNVISGIP IYRVMDRQGQIINP SED PHL PQEEVLKFYRSMT

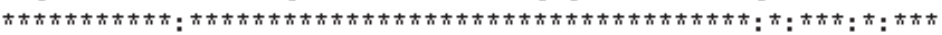

LLNTMDRIL YESQRQGRIS FYMTWYGEEGTHVGSAAALDDTD LVFGQYREAGVLMYRDYP L LNTMDRI L YE SQRQGRI S FYMTNYGEEGTHVGSARALDDTD LVFGQYREAGVLMYRDYP L LNTMDRI L YE SQRQGRIS FYMTNYGEEGTHVGSAДALDNTD LVFGQYREAGVLMYRDYP LLNTMDR I Y SE SQRQGRIS FYMTNY GEE GTHVGSARALDNTD LVFGQYREA GVLMYRDYP LLNTMDR I Y YE SQRQGRIS FYMTNYGEE GTHVGSARALDNTD LVFGQYREAGVLMYRDYP LLNTMDRI L YE SQREGRIS FYMTNYGEEGTHVGSAдДALERTD LVFGQYREA AVVLMYRDYP

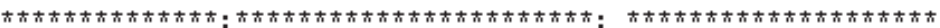

LEL FMAQCYGNVSD LGKGRQMPVHYGCRERHFVTISSP LATQIPQAVGAAYAAKRANANR LE L FMAQCYGNVSD L GKGRQMPVHYGCRERHFVTISSP LATQIPQAVGAAYAAKRANANR LEL FMAQCYGNISD L GKGRQMPVHYGCKERHFVTISSP LATQIPQAVGAHYAAKRANANR LEL FMAQCYGNISD LGKGRQMPVHYGCKERHFVTISSP LATQIPQAVGAAYAAKRANANR LEL FMAQCYGNISD L GKGRQMPVHYGCKERHFVTISSP LATQQIPQAVGAAYAAKRANANR LE L FMSQCYGNVND P GKGRQMPVHYGCKERHFVTISSP LATQIPQAVGAAYAAKRANANR

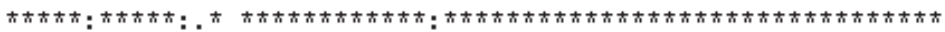

WICYFGE GÄДSEGDAHAGFNFAДRLECPIIFFCRNNGYAISTPTSEQYRGDGIAARRGPG WVICYFGEGÄASEGDAHAGFNFÄATLECPIIFFCRNNGYAISTPTSEQYRGDGIÄARGPG VICYFGEGÄSEGDAHÄGFNFÄTLECPIIFFCRNNGYAISTPTSEQYRGDGIAARGPG WICYFGEGAASE GDAHAGFNFAATLECPIIFFCRMNGYAISTPTSEQYRGD GIAARGPG WICYFGE GÄĂSEGDAHAGGFFAДALECPIIFFCRNNGYAISTPTSEQYRGDGIÄARGPG IVICYFGE GAASE GDAHAGFNFAATLECPIIFFCRMNGYAISTPTSEQYRGDGIAARGPG

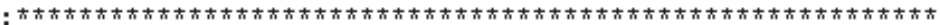

YGILSIRVD GNDVFAVYNATKEÅRRAVAENQP FLIEAMTYRIGHHSTSDDSSAYRSVDE YGILSIRVD GNDVFAVYNATKEARRRAVAENQP FLIEAMTYRIGHHSTSDDSSAYRSVDE YGIMSIRVD GNDVFAVYNATKEARRRAVAENQP FLIEAMTYRIGHHSTSDDSSAYRSVDE YGIMSIRVD GNDVFĂVYNĂTKEÅRRRAVAENQP FLIEAMTYRIGHHSTSDDSSAYRSVDE YGIMSIRVD GNDVFAVYNATKEARRRAVAENQP FL IEAMTYRI GHHSTSDD SSAYRSVDE YGIKSIRVD GNDVFAVYNATKEARRRAVAENQP FLIEAMTYRIGHHSTSDDSSAYRSVDE

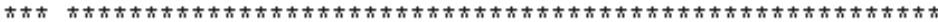

VNYWDKQDHP ISRLRHHLQSRGTWDDEQEKAWRKQSRKKWMEÄFEQÄERKLKPNPNLIFS VNYTDKQDHP ISRLRHHLQSRGUTDDEQEKAINKQSRKKVMEAFEQAERKLKPNPSLIFS VNYWDKQDHPVSRLRHYL LSQGUTDDEEQEKAITRKQSRKKVMEA FEQÄERKPKPNPNLLFS VNYWDKQDHP ISRLRHYL LSQGJTDDEEQKAJTKQSRRKWMEAFEQÄERKPKPNPNLLFS VNYWDKQDHP ISRLRHYL LSQGUTWDEEQEAHRKQSRKKVMKAFEQAERKPKPNPNLLFS VNYWDKQDHP ISRLRQYL LNQGUTWDEEQEKAJRKQSRKKVMEAFEQAERKLKPNPSLLFS

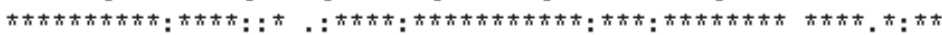

DVYQEMPAQLRKQQESLARHLQTYGEHYPLDHFEK DVYQEMPAQLRKQQESLÄRHLQTYGEHYPLDHFEK DVYQEMPAQLRKQQESLARHLQTYGEHYPLDHFDK DVYQEMPAQLRKQQESLÄRHLQTYGEHYPLDHFDK DVYQEMPAQLRKQQESLARHLQTYGEHYPLEHFDK DVYQEMPAQLRRQQESLARHLQTYGEHYPLDHFEK

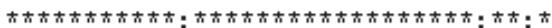

Fig. 2: The alignment of the protein encoded by sheep BCKDHA gene and five other kindsof BCKDHA. 
Sheep

Bovine

Human

Mouse

Rat

Chicken

Sheep

Bovine

Human

Mouse

Rat

Chicken

Sheep

Bovine

Human

Mouse

Rat

Chicken

Sheep

Bovine

Human

Mouse

Rat

Chicken

Sheep

Bovine

Human

Mouse

Rat

Chicken

Sheep

Bovine

Human

Mouse

Rat

Chicken

Sheep

Bovine

Human

Mouse

Rat

Chicken

Sheep

Bovine

Human

Mouse

Rat

Chicken
MLLKTVLL LALVAQVL VLENGL LRKP PMGULÄWER FRCNIDCNED PKSCISEE L FMEMAD MLLKTVLL LALASQVLVLENGLLRKP PMGWLALERFRCNIDCSED PKNCISEQL FMEMAD ML LKTVLL L GHVAQVLMLDNGLLQTP PMGWLALERFRCNINCDED PKNC ISEQL FMEMAD MLQKTVL L LAL VAQVLMLENGL LRTP PMGWLATER FRCNIDCVED PKNC ISERL FMEMAD MLQKTVL L LALVAQVLMLENGL LRTP PMGWLÄLERFRCNINCEED PKNC ISERL FMEMAD -LENGLARTP PMGWLALIERFRCNWNCRED PRQCISEML FMEMAD

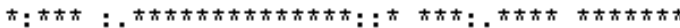

RLAQDGURE LGYVLNIDDCUIGG-RDAKGNLVPDRKRFPHGIAFLÄDYAHSLGLKLGIY RLAODGWRDLGYVYLNIDDCWIGG-RDAKGNLVPDRKRFPHGIAFLADYAHSLGLKLGIY RMAQDGWRDMGYTYLNIDDCWIGG-RDASGRLMPD PKRFPHGIP FLADYYHSLGLKLGIY RLAQDGWRD LGYYLNIDDCWIGG-RDASGRLIPD PKRFPHGIAFLADYAHSLGLKLGIY RLAQDGWRD LGYVLNIDDCWIGG-RDATGRLIPD PKRFPHGIAFLADYAHSLGLKLGIY RIÄED GURE LGYKYINIDDCWAAKORDAEGRLVPD PERFPRGIKALADYVHARGLKLDIY

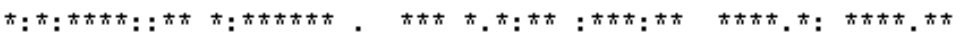

EDLGNFTCMGYPGTTLDKWVDAOTFAETKVDMLKLDGCYSSPQERAEGYPKMAДALLNAT EDLGNFTCMGY PGTTLDKWVDARQTFÄEUKVDMLKLDGCYSTPQERAEGYPKMAДĂLNÄT ADMGNFTCMGYP GTTLDKWVDAOTFÄEUKVDMLKLDGCFSTPEERAQGYPKMAДĂLNATT EDMGKMTCMGYP GTTLDKVE LDAETF ÄEUKVDMLKLDGCFSSSRERÄGYPKMÄÄLLNAT EDMGKMTCMGY GTTLDKVE LDAATF AELTKVDMLKLDGCYSTPKERAE GYPKMAДALNAT GDLGRLTCGGYPGTTLDRVEQDAQTFAEUGVDMLKLDGCYSSGKEQAQGYPQMARALNST

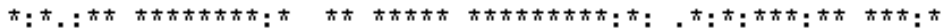

GRP IAFSCSWPAYEGGL P PKVNTLLAD ICNLWRNFDD IQDSWRSVLS ILDWFVAHQDVL GRP IAFSCSWPAYEGGL P PKVNYTL LAD ICNLWRNFDD IQDSWRSVLSVLDWFVTHQDVL GRP IAFSCSWPAYE GGL P PRVNY L LAD I CNLWRWYDD IQD SUTJSVL S I LNWFVEHQD IL GRP IAFSCSWPAYEGGL P PKVNYTV VSRVWLWRWYKD IQDSWKSVL S ILDWFVRHQDVL GRP IAFSCSWPAYEGGL P PKVNYTEVAGTCNLWRNYKD IQD SWKSVL S ILDWFVKHQD IL GRP IVYSCSWPAYQGGL P PKVNYTLLGEICNLWRNYD IQDSWD SVLSIVDWFFTNQDVL

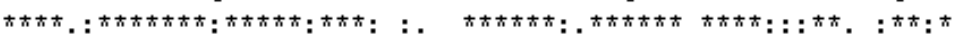

QPVAGP GHTND PDVLL IGNFGLSFEQARAQMALWTVLÄAP L LMSTD LRTI SAONDD ILQN QP IAGP GHTND PDML L I GNFGL S FEQAQAQMALWTVLAДP L FMSTD LRTI SAQMND ILQN QPVAGP GHTND PDML L I GNFGL S LEQSRAQMALWTVLABP L LMSTD LRTISAQNMD ILQN QPVAGP GHTND PDML L I GNFGL S FDESRAQMALWTVLÄAP L LMSTD LRTIS PQMN ILQN QPVSGP GHTND PDML L I GNFGLS FDESRAQMALWTVLAA P L FMSTD LRTISPQNID ILQN QPFAGPGHTND PDML I IGNFGLSYEQSRSQMALWTIMÄAP L LMSTD LRTI SPSAKKILQN

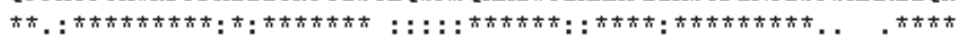

PLMIKINQDPLGIQGRRI LKEKSYIEVYLRP LVNEẢSÄIVFFSRRTDMPYHYHSSLARLN PLMIKINQD P LGIQGRRILKEKSHIEVYLRP LASEA SAIVFFSRRMDMPYHYHS SLARLN PLMIKINQD P LGIQGRRIHKEKS LIEVMRP LSNKA SAL VFFSCRTDMPYRYHSSLGQLN PLMIKINQD PLGIQGRRI LKSKSHIEVFKRYLSNQASALLVFFSRTDMP FRFHCSL LELN PLLIKINQD PLGIQGRLIFKSKSHIEVFKRNLSDDASALVFFSRRTDMPYHFHC SLLELN RLMIQINQD P LGIQGRRIIKE GSHIEVFLRP LSQAASALVFFSRRTDMP FRYTTSLAKLG

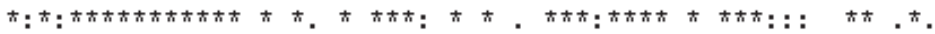

FSSSWVEADDVYTGEIISGLQDKTNFTVIINPSGWWMTY YP ISKLGIPQQ-------FSSSWVEAQDWTGD IISGLQDKTNFTVIINPSGWNTYL YP IRKLEIPQQ-------FTGSVIYEAQDVYSGD I ISGLRDETNFTVI INP SGVNMTYLYP IKNLEMSQQ-------YPKGRVYE GQNVFTGD IFSGLQTEVNFTVI INPSGVWMTLYP IKD LGISTMMSHJ---YPKGSVYGQNVFTGD IISGLHPETNFTVIINPSGWNTIYLYPVKGLGIYTMMSQL---FPMGAAYEVQDVYSGKIISGLKTGDNFTVIINPSGVWMTLCPKALLIQQQAPGGPSRLP

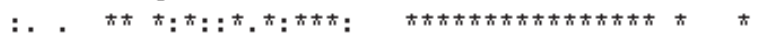

$--$

$--$

$--$

$--$

$--$

LL

Fig. 3: The alignment of the protein encoded by sheep NAGA gene and five other kinds of NAGA. 
Sheep

Bovine

Human

Mouse

Rat

Chicken

Sheep

Bovine

Human

Mouse

Rat

Chicken

Sheep

Bovine

Human

Mouse

Rat

Chicken

Sheep

Bovine

Human

Mouse

Rat

Chicken

sheep

Bovine

Human

Mouse

Rat

Chicken

Sheep

Bovine

Human

Mouse

Rat

Chicken

Sheep

Bovine

Human

Mouse

Rat

Chicken

Sheep

Bovine

Human

Mouse

Rat

Chicken
MLLKTVLLLAL VAQVL VLENGL LRKP PMGWLATERFRCNIDCNED PKSCISEE L FMEMAD MLLKTVLLLALASQVLVLENGL LRKP PMGWLATERFRCNIDC SED PKNC ISEQL FMEMAD MLLKTVLLLGHVAOVLMLDNGLLQTP PMGWLAWERFRCNINCDED PKNCISEQL FMEMAD MLQKTVLL LALLVAQVLMLENGL LRTP PMGWLALER FRCNIDCVED PKNC ISERL FMEMAD MLQKTVLLLALVAOVLMLENGLLRTP PMGWLAWERFRCNINCEED PKNC ISERL FMEMAD -----------LENGLARTP PMGWLAWERFRCWVNCRED PRQCISEML FMEMAD

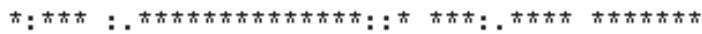

RLÅDGURE LGYVLNIDDCWIGG-RDAKGNL VPDRKRFPHGIẢFLÄDYAHSLGLKLGIY RLAQDGURD LGYVLNIDDCUIGG-RDAKGNLVPDRKRFPHGIAFLADYAHSLGLKLGIY RMAQD GJRDMGYTYLNIDDCWIGG-RDASGRLMPD PKRFPHGIP FLADYVHSLGLKLGIY RLAQDGURDLGYVLNIDDCWIGG-RDASGRLI PD PKRFPHGIAFLADYAHSLGLKLGIY RLAQDGURDLGYVLNIDDCWIGG-RDATGRLIPDPKRFPHGIAFLADYAHSLGLKLGIY RIAEDGURE LGYKYINIDDCWAДKQRDÄEGRLVPD PERFPRGIKALADYWHARGLKLDIY

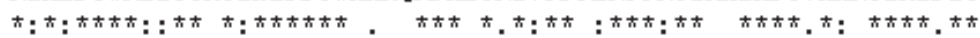

EDLGNFTCMGYPGTTLDKWVDAQTFAEUKVDMLKLDGCYSSPQERAEGYPKMAДALNAT EDLGNFTCMGYPGTTLDKVVDDAOTFAEUKVDMLKLDGCYSTPQERAEGYPKMAAALNAT ADMGNFTCMGYPGTTLDKWWQDAQTFAEUKVDMLKLDGCFSTPEERAQGYPKMẢẢLLNAT EDMGKMTCMGYPGTTLDKVE LDAETFAEUKVDMLKLD GCFSSSRERAEGYPKMAДALNAT EDMGKMTCMGYPGTTLDKVE LDÄATFÄEUKVDMLKLD GCYSTPKERAEGYPKMAдДALNÄT GDLGRLTCGGYPGTTLDRVEQDAQTFAEUGVDMLKLDGCYSSGKEQĀQGYPQMARALNST

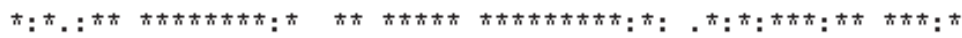

GRP IAFSCSWPAYEGGL P PKVNYTL LAD ICNLWRNFDD IQDSWRSVL SILDWFVAHQDVL GRP IAFSC SWPAYEGGL P PKVNYTL LAD ICNLWRNFDD IQD SWRSVL SVLDWFVTHQDVL GRP IAFSCSWPAYEGGL P PRVNY L LAD ICNLWRWYD IQD STJSVL I ILNJWVHQD I L GRP IAFSCSWPAYEGGL P PKVNYTEVSRVCNLWRWYKD IQD SWKSVL I ILDWFVRHQDVL GRP IAFSCSWPAYEGGL P PKVNYTEVAGTCNLWRWYKD IQD SWKSVL I ILDWFVKHDD IL GRP IVYSCSWPAYQGGL P PKVNYTL LEE ICNLWRNYD IQD SWDSVLSIVDWFFTNQDVL

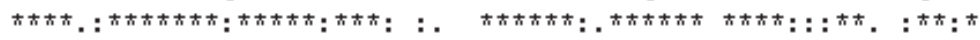

QPVAGP GHTND PDVLL IGNFGLSFEQARAQMALWTVLĂAP L LMSTD LRTISAONVD ILQN QP IAGP GHTND PDML L I GNFGL SFEQAQA QMAL WTVLÄAP L FMSTD LRTI SAOMM ILQN QPVAGP GHTND PDML L I GNFGL S LEQSRAOMAL WTVLÄẢP L LMSTD LRTI SAOMN ILQN

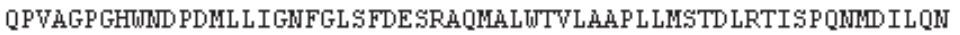
QPVSGP GHTND PDML L I GNFGLS FDE SRAQMALWTVLÄAP L FMSTD LRTISPQNID ILQN QPFAGP GHTIND PDML I IGNFGLSYEQSRSQMAL WTIMAAP L LMSTD LRTISP SAKKILQN

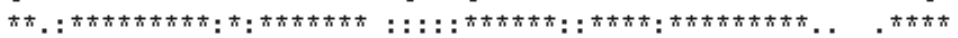

PLMIKINQDPLGIQGRRI LKEKSYIEVYLRP LVNEAૂSĂIVFFSRRTDMPYHYHSSLÄRLN PLMIKINQD P LGIQGRRI LKEKSHIEVYLRP LÅSEASAAIVFFSRRMDMPYHYHSSLARRLN PLMIKINQD PLGIQGRRIHKEKS L IEVMRP LSMKASA L VFFSCRTDMPYRYHSSLGQLN PLMIKINQDPLGIQGRRILKSKSHIEVFKRYLSNQÄSALLVFFSRRTDMP FRFHCSLLELN PLLIKINQD P LGIQGRL I FKSKSHIEVFKRNL SDDASSẢLVFFSRRTDMPYHFHCSL LELN RLMIQINQD P LGIQGRRI IKE GSHIEVFLRP LSQAASALVFFSRRTDMP FRYTTSLAKLG

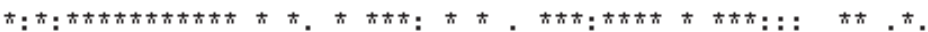

FSSSWVEAQDVYTEEIISGLQDKTNFTVIINPSGWNTYLYPISKLGIPQQ-------FSSSVVEAQDVYTGDIISGLQDKTNFTVIINPSGVVMTYLYP IRKLEIPQQ-------FTGSVIYEAODVYSGD IISGLRDETNFTVIINPSGWWMY LYP IKNLEMSQQ-------YPKGRVYEGQNVFTGD IFSGLQTEVNFTVIINPSGWNTTY L YP IKD LGISTMMSHT---YPKGSVYEGQNVFTGD IISGLHPETNFTVIINPSGWNTWY YPVKGL GIYTMMSQL---FPMGAHYEVQDVYSGKIISGLKTGDNFTVIINPSGVWMINLCPKALLIQQQAPGGPSRLP

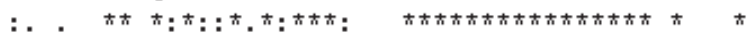

--
--
--
--
--
LL

Fig. 4: The alignment of the protein encoded by sheep HEXA gene and five other kinds of HEXA. 
(http://www.ncbi.nlm.nih.gov/BLAST) revealed that these genes were not homologous to any of the known sheep genes and they were then deposited into the GenBank database (Accession number: EU579456, EU579460 and EU579459). The sequence prediction was carried out using the GenScan software and results showed that the 1344bp, 1236bp and 1590bp cDNA sequences represent three single genes, which encoded 447,411,529 amino acids, respectively. Finally these three novel sheep genes were assigned to GeneIDs: 100145857,100145858 and 100145856.

Further BLAST analysis of these proteins revealed that the sheep BCKDHA has high homology with the Branched chain keto acid dehydrogenase E1, alpha polypeptide BCKDHA of five species: chimpanzee $(93 \%)$, human $(96 \%)$, crabeating macaque $(93 \%)$, bovine $(98 \%)$ and mouse $(91 \%)$. The sheep NAGA gene has high homology with the alpha-Nacetylgalactosaminidase(NAGA) of five species-human $(85 \%)$, bovine $(94 \%)$, mouse $(91 \%)$, rat $(83 \%)$, and chicken $(74 \%)$. The sheep HEXA has high homology with the hexosaminidase A HEXA of five speciescattle (98\%), human (84\%), Bornean orangutan $(84 \%)$, rat $(80 \%)$, and mouse $(81 \%)$.

Based on the results of the alignment of BCKDHA, NAGA and HEXA, the phylogenetic trees were constructed using the Dendrogram procedure of ClustalW software (http://align.genome.jp/), as shown in Fig. 5, Fig. 6, Fig. 7.

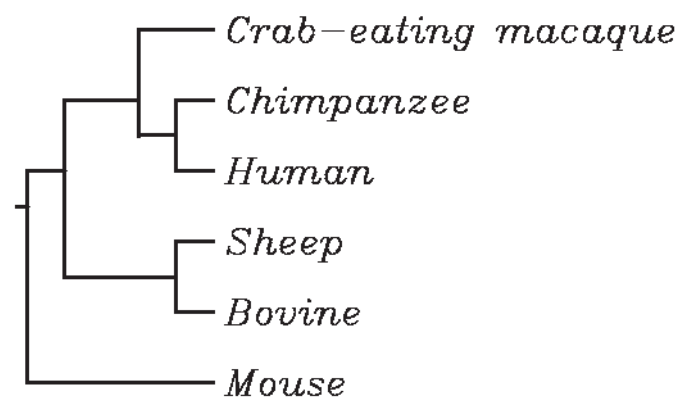

Fig. 5: The phylogenetic tree for seven kinds of BCKDHA.

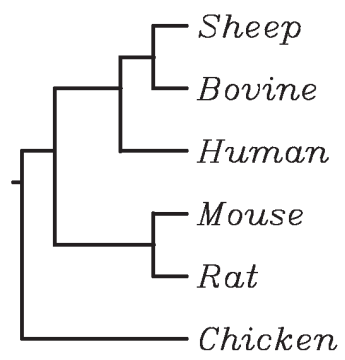

Fig. 6: The phylogenetic tree for six kinds of NAGA.

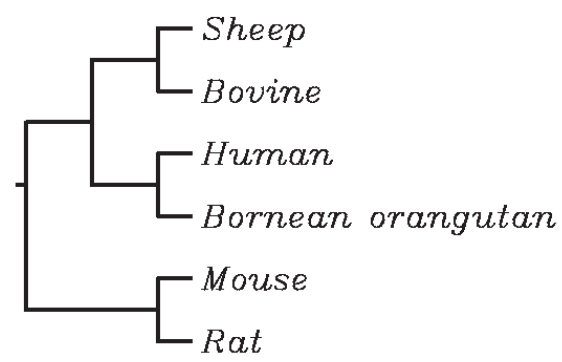

Fig. 7: The phylogenetic tree for six kinds of HEXA.

The phylogenetic tree analysis revealed that the sheep BCKDHA, NAGA, and HEXA all have closer genetic relationships to the BCKDHA, NAGA, and HEXA of the bovine.

\section{Tissue expression profile}

Tissue expression profile analysis was carried out and results revealed that the sheep BCKDHA gene was moderately expressed in the liver, large intestine, lung and muscle, weakly expressed in the small intestine, kidney, fat and spleen. The sheep NAGA gene was highly expressed in the large intestine, moderately in muscle and the spleen, and weakly expressed in the liver, small intestine, lungs, fat, and the kidney. The sheep HEXA gene was highly expressed in fat, muscle and the kidney, moderately expressed in the liver, small intestine and spleen, and hardly expressed in the large intestine and lung (Fig. 8).

Chinsky (1998) reported the presentation of maple syrup urine disease associated with a nonsense mutation (R242X) in coding region of BCKDHA gene. To NAGA gene, mutation p.D217N 
(c.649G $>\mathrm{A}$ ) in exon 6, mutation p.E325K (c.973G $>A$ ) in exon 8, mutations of R329Q and $\mathrm{R} 329 \mathrm{~W}$, may have roles in alpha-Nacetylgalactosaminidase deficiency with cardiomyopathy (Chabás et al., 2007; Kanekura et al., 2005). For HEXA gene, a substitution of cytosine 1351 by guanosine $(\mathrm{C} 1351 \mathrm{G})$ and other mutations have been also reported to be associated with TaySachs disease (Karpati et al., 2004; McGinniss et al., 2002). Therefore, isolation of the encoding regions of the sheep BCKDHA, NAGA and HEXA genes is utmost important to detect these three kinds of diseases.

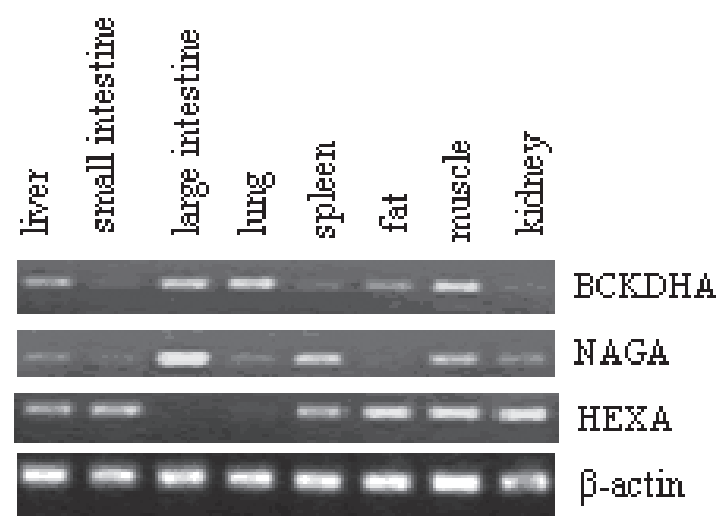

Fig. 8: Tissue expression distribution of sheep BCKDHA, NAGA, and HEXA genes.

From the alignment analyses of the BCKDHA, NAGA and HEXA proteins, there were no differences found at the above loci in humans, sheep, mice and other mammals, but many other amino acid differences were found in these three kinds of proteins. This implies that in future research of these three genes, attention should be given to whether these differences in amino acids and reported human mutations also occur in sheep and the potential associations of them to sheep diseases.

From the phylogenetic tree analysis, we found that sheep BCKDHA, NAGA and HEXA genes have much closer relationships to human than to mouse genes. This suggests that sheep would be more suitable as a model animal to study these three kinds of human mutation diseases. In other words, theoretically, sheep are also animals susceptible to these diseases.

We also noted that human and mouse BCKDHA, NAGA and HEXA genes are expressed in most tissues (http:// www.ncbi.nlm.nih.gov/UniGene). From the tissue distribution analysis in our experiment it can be seen that these genes were obviously differentially expressed in some tissues and there was no expression in some tissues. As we have not yet studied functions or protein levels, there might be many possible reasons for differential expression of these three sheep genes. The best explanation for this under current conditions is that the biological activities related to the mRNA expression of these genes were presented differently in different tissues.

The mutations of these three human or mouse genes at some loci are related to three types of important inherited diseases to which sheep are also susceptible. Thus, detection of the mutations of these three sheep genes and the association of these potential mutations with corresponding sheep diseases will be of interest beyond the importance to sheep production. Therefore, more research based on these preliminary foundations is needed.

\section{ACKNOWLEDGEMENTS}

This work is supported by grants from the National Natural Science Foundation of China (No. 30800810) and the National Basic Research Program of China (973 Project) (2007CB116201).

\section{REFERENCES}

CHABÁS A, DUQUE J, GORT L. A new infantile case of alpha-N-acetylgalactosaminidase deficiency. Cardiomyopathy as a presenting symptom. J Inherit Metab Dis. 2007 Feb; 30(1): 108

CHINSKY J, APPEL M, ALMASHANU S, COSTEAS P, AMBULOS N JR, CARMI R. A nonsense mutation $(\mathrm{R} 242 \mathrm{X})$ in the branched-chain alpha-keto acid dehydrogenase E1 alpha subunit gene (BCKDHA) as a cause of maple syrup urine disease. Hum Mutat. 1998, 12(2): 136

FLASCHKER N, FEYEN O, FEND S, SIMON E, SCHADEWALDT P, WENDEL U. Description of the 
mutations in 15 subjects with variant forms of maple syrup urine disease. J Inherit Metab Dis. 2007, 30(6): 903-9

HARDISON RC. Comparative genomics. PLoS Biol. 2003,1(2): E58

HERRMANN T, SCHINDLER D, TABE H, ONODERA O, IGARASHI S, POLACK A, ZEHNPFENNIG D, SHOJI TSUJI. Molecular cloning, structural organization, sequence, chromosomal assignment, and expression of the mouse alpha-N-acetylgalactosaminidase gene. Gene. 1998 May 12; 211(2): 205-14

KANEKURA T, SAKURABA H, MATSUZAWA F, AIKAWA S, DOI H, HIRABAYASHI Y, YOSHII N, FUKUSHIGE T, KANZAKI T. Three dimensional structural studies of alpha-N-acetylgalactosaminidase (alpha-NAGA) in alpha-NAGA deficiency (Kanzaki disease): different gene mutations cause peculiar structural changes in alpha-NAGAs resulting in different substrate specificities and clinical phenotypes. J Dermatol Sci. 2005 Jan; 37(1): 15-20

KARPATI M, GAZIT E, GOLDMAN B, FRISCH A, COLOMBO R, PELEG L. Specific mutations in the HEXA gene among Iraqi Jewish Tay-Sachs disease carriers: dating of founder ancestor. Neurogenetics. $2004 \mathrm{Feb}$; 5(1): 35-40

LIU GY, XIONG YZ.Isolation, sequence analysis and expression profile of a novel sheep gene, NIP7, differentially expressed in the Longissimus dorsi muscle tissues from Meishan, Meishan x Large White cross and Large White sheeps.Mol Biol Rep. 2007, 344: 213-219

LIU YG, XIONG YZ, DENG CY, ZUO B, ZHANG JH. Comparison of gene expression patterns in Longissimus dorsi of sheeps between the high-parent heterosis cross combination Landrace $\backslash$ Large White and the mid-parent heterosis cross combination Large White $\$ Meishan. Asian-Aust J Anim Sci. 2004,17(9): 1192-1196

MCGINNISS MJ, BROWN DH, FULWILER A, MARTEN M, LIM-STEELE JS, KABACK MM. Eight novel mutations in the HEXA gene. Genet Med. 2002 MayJun; 4(3): 158-61

MIKLYAEVA EI, DONG W, BUREAU A, FATTAHIE R, XU Y, SU M, FICK GH, HUANG JQ, IGDOURA S, HANAI N, GRAVEL RA.Late onset Tay-Sachs disease in mice with targeted disruption of the Hexa gene: behavioral changes and pathology of the central nervous system.Brain Res. 2004 Mar 19; 1001(1-2): $37-50$

NAIK MT, HUANG TH. Conformational stability and thermodynamic characterization of the lipoic acid bearing domain of human mitochondrial branched chain alpha-ketoacid dehydrogenase. Protein Sci. 2004,13(9): 2483-92

PESSOA-PUREUR R, WAJNER M. Cytoskeleton as a potential target in the neuropathology of maple syrup urine disease: insight from animal studies.J Inherit Metab Dis. 2007,30(5):664-72. 